\title{
Role of a multidisciplinary program in improving outcomes in cognitively impaired heart failure older patients
}

\section{Efficacia di un programma multidisciplinare in pazienti con scompenso cardiaco e deficit cognitivo}

\author{
Donatella Del Sindaco1, Giovanni Pulignano2, Andrea Di Lenarda3, \\ Luigi Tarantini4, Giovanni Cioffi5, Stefano Tolone2, Maria Denitza Tinti2, \\ Luca Monzo2, Giulia Barbati6, Giovanni Minardi2
}

\begin{abstract}
Role of a multidisciplinary program in improving outcomes in cognitively impaired heart failure older patients. D. Del Sindaco, G. Pulignano, A. Di Lenarda, L. Tarantini, G. Cioffi, S. Tolone, M.D. Tinti, L. Monzo, G. Barbati, G. Minardi.

Background: Cognitive impairment (CI) frequently complicates Heart failure (HF) and is associated with increased mortality and morbidity. Previous studies reported that nurse-lead home-based multidisciplinary program (MP) may not improve the prognosis of this high-risk group. In the present study, we analysed the relative effectiveness of an integrated hospital-based MP in patients with cognitive impairment.

Methods: Consecutive ( $n=173)$ community-living outpatients aged $>70$ years (mean $77+6,48 \%$ women) randomized to a MP $(n=86)$ or usual care (UC) $(n=87)$ were enrolled in stable clinical conditions. Cognitive status was assessed by means of Folstein Mini Mental State Examination (MMSE).

Results: CI (MMSE $\leq 24)$ was present in $41.6 \%(42,5 \%$ UC vs $40.7 \% \mathrm{MP} p=n s)$. The variables independently associated to $C$ I were: older age, education level $<5$ years, anemia and severe
\end{abstract}

renal dysfunction. During a 2-year follow-up, 59 patients died $(31.4 \%)$ with no significant difference between intervention group. At multivariate analysis, in the entire cohort, CI was independently associated to death (HR 2,077[95\% CI 1,097$3,931])$, HF admissions $(2,133[1,346-3,381])$, death/HF admissions $(1,784[1,132-2,811])$ and all-cause admissions $(1,473[1,008-2,153]$. When considered according to intervention groups, CI was independently associated to all-cause death $(3,603$ [1,553-8,358], death/HF admissions $(2,029[1,200-3,432])$ and $\mathrm{HF}$ admissions $(2,474[1,406-4,353])$ but not to all-cause admissions. The assignment of patients with CI to MP was associated to a significant reduction in $\mathrm{HF}$ admissions vs $\mathrm{UC}$ $(0,503[0,253-0,999]$ (all interaction tests $\mathrm{p}=\mathrm{ns}$ ).

Conclusions: This study suggests that $\mathrm{CI}$ is very common and associated to worse prognosis in heart failure and that hospital-based MP seems to improve outcomes in these patients through reduction of heart failure hospital admission.

Keywords: heart failure, disease management, cognitive impairment, elderly, prognosis.

Monaldi Arch Chest Dis 2012; 78: 20-28.

${ }^{1}$ Heart Failure Unit. Division of Cardiology, INRCA Institute of Care and Research for Elderly, Rome, Italy.

2 Heart Failure Clinic. Division of Cardiology/C.C.U. San Camillo Hospital, Rome, Italy.

${ }_{3}^{3}$ Cardiovascular Center and University, Trieste, Italy.

${ }^{4}$ Heart Failure Clinic. Division of Cardiology, San Martino Hospital, Belluno, Italy.

5 Heart Failure Clinic. Division of Cardiology, Villa Bianca Hospital, Trento. Italy.

6 Department of Medical Sciences, University of Trieste, Italy.

Supported in part by the non-profit organization ADRIANO - Italian Association for Research on Cardiac Disease in Older Patients (Program AD-002-2005).

Corresponding author: Giovanni Pulignano, MD; Via G. Livraghi 1; I-00152 Rome, Italy; Tel: ++390658704562; Fax: ++39065815205; E-mail address: gipulig@yahoo.it

\section{Background}

Despite the availability of effective treatments, management of older heart failure (HF) patients remains sub-optimal with frequent hospital admissions, disability and high mortality rates [1-3]. Older patients include a heterogeneous population in which the clinical status and subsequent risk profile result from a complex interaction between different domains: age-related cardiovascular changes, car- diovascular disease, co-morbidities (not only hypertension, diabetes, renal failure, chronic obstructive pulmonary disease and anemia, but also cognitive impairment, arthritis, incontinence, psychological problems, limitations in activities of daily living and social issues $[4,5]$.

The management of the patient with HF is therefore complex and patients are asked to make lifestyle changes, take multiple drugs, and are exposed increasingly to device therapy. In this per- 
spective, disease management programs have been developed and found to be effective in improving outcomes [6-8]. However, important questions are still open, including the most appropriate patient selection process, the optimal follow-up intensity and duration and, finally, whether such interventions are cost-effective in different subgroups [9, 10].

Cognitive impairment frequently complicates heart failure with deficits being most prominent in the domains of executive function, memory, attention, language, and mental speed and is associated with disability, morbidity and mortality [11-13]. Even mild forms of cognitive impairment may seriously interfere with HF management and self-care through impaired quality of life, decision-making and ability to adhere to complex medication and dietary regimens [14]. In order to improve outcomes in these patients, an approach to enhance adherence to care plan and specific behaviours should be considered. Previous studies reported that nurse-lead home-based multidisciplinary program (MP) may not improve the prognosis of this high-risk group [15]. In this sub-analysis of a previously published randomized study comparing MP and usual care (UC) [15], we investigated: 1) the influence of cognitive impairment on outcome of older HF patient and, 2) if a MP represents an appropriate model of care in these patients.

\section{Methods}

Study design: The study was conducted at two hospital HF clinics and primary care facilities. We evaluated consecutive HF patients aged 70 years or more, both males and females, with reduced and normal left ventricular Ejection Fraction and discharged home after a hospitalization due to New York Heart Association (NYHA) functional class III/IV HF, requiring intravenous diuretic and/or inotropes or vasodilators therapy [16]. The diagnosis was determined according to the European Society of Cardiology guidelines [17]. Patients were excluded if they had: 1) valvular heart disease requiring planned surgery, 2) active substance abuse, severe gait impairment, severe dementia and/or MMSE $<11$ or psychiatric disease, 3) coexisting non-cardiac illness likely to reduce life expectancy, 4) need for long-term intravenous inotrope therapy, 5) unwillingness to provide informed consent, and 6) living in a nursing home or outside the area served by the clinical sites. The protocol is consistent with the Principles of the Declaration of Helsinki and all participants gave their informed consent. No Institutional Review Board approval for this type of study (a non pharmacological trial) was required for at the time of study in our Institution. Details on randomisation and blinding procedures and on the MP intervention have been previously reported [16] (Figure 1). The MP combined outpatient hospital-based and primary care. In each of the two participating teams, the members were a cardiologist, specialized nurses and primary care physicians. The components of the MP were: discharge planning, patient and caregiver education, therapy optimization, improved communication and early attention to signs and symptoms. Intensive follow-up was based on scheduled and unplanned hospital visits, nurse's phone call and office and home primary care physician visits. Patients assigned to UC received all treatments and services ordered by their primary care physician and/or cardiologist or internist. The follow-up was based on phone calls (to the patient and/or family members or primary care physician) after one, three months from hospital discharge, and thereafter every six months.

Clinical evaluation and definitions. Initial assessment included history, physical examination, NYHA class, 12-lead electrocardiogram, Dopplerechocardiography and a multidimensional assessment performed using previously validated instruments that explored the following areas: 1) Socioeconomic: years of education, marital status, living arrangement and financial income; 2) ability to perform basic (BADL) and instrumental (IADL) activities of daily living, using two modified 7-item scales [18, 19]; 3) cognitive function, measured with Folstein's Mini Mental State Examination (MMSE) (with items measuring orientation, registration, attention and calculation, recall, language and visual spatial ability), [20]; 4) depressive symptoms, measured with the 15-item Geriatric Depression Scale (GDS) [21]; 5) Charlson Comorbidity index [22]. Cognitive impairment was defined by a Mini Mental State Examination score less or equal than 24. Physical disability was defined as need of assistance in performing at least one basic or two instrumental Activities of Daily Living.

Outcome measures. Planned outcome variables were all-cause deaths, all-cause and HF-related admissions the composite of death from any cause and/or hospital admissions for $\mathrm{HF}$ and total costs of care. All patients were followed for two years and clinical status, medications, number of primary care and specialist visits and events were recorded at each visit or phone call. Events were collected also using phone calls, discharge reports, hospital and administrative databases, death certificates and were blindly evaluated by a central endpoint committee composed of three cardiologists, who had no knowledge of the randomization group assignment. All questionnaires were analyzed by researchers blinded to intervention group assignment.

Estimation of costs. In this study the National Health System (NHS) perspective was adopted, as in Italy it provides all health care services for patients affected by moderate-to-severe HF. Data on resource utilization of MP compared to UC have been previously published [16]. Costs were collected prospectively and only comprised direct costs (pre-discharge education and assessment, medications, hospital and home visits, echocardiography and hospitalizations) calculated on the basis of NHS charges at the time of the study and stratified according to the presence or absence of cognitive impairment.

Statistical analysis. Groups were compared by the $t$ test for normally distributed continuous variables and expressed as means \pm standard deviations (SDs), the chi square test and Fisher exact test for 


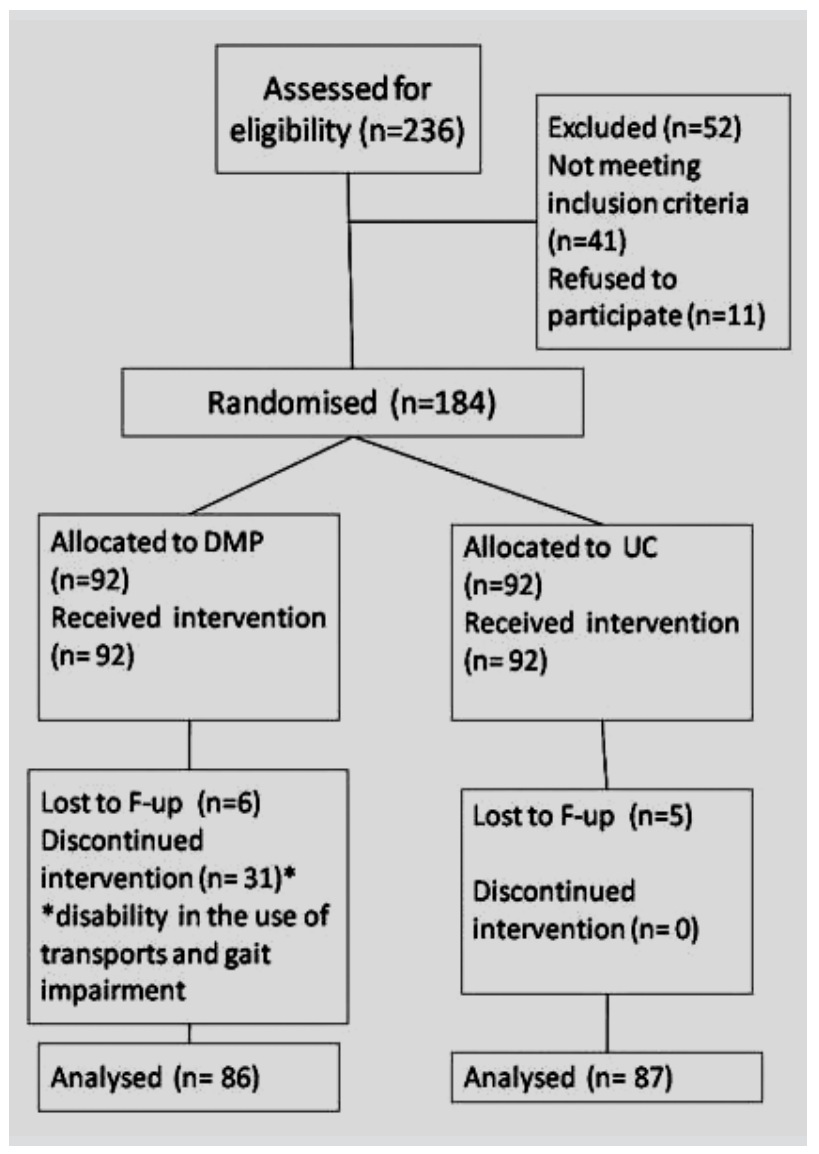

Figure 1. Diagram showing the flow of participants through each stage of the trial.

categorical variables (expressed as counts and percentages). Logistic regression analysis was performed in order to identify factors potentially related to cognitive impairment (MMSE score $\leq 24$ ) with calculation of adjusted odds ratios (OR) and 95\% confidence intervals (CIs). Outcome analysis was conducted according to an intention-to-treat approach. Multivariable Cox proportional-hazards models with adjustment for potentially confounding variables were used to estimate the risk of outcomes. Differences in treatment effects according to cognitive groups were evaluated by tests of interaction and the statistical significance was determined by a Wald chi-square test for interaction. Four KaplanMeier stratified survival curves were then generated to compare - using Mantel-Cox log rank test - the event-free survival of patients with and without cognitive impairment in the usual care group and MP group. Data for all event-free patients were censored on study day 730 . A p value less than 0.05 was considered significant. Analyses were performed using SPSS for Windows 13.01 (SPSS Inc. USA).

\section{Results}

During a two-year period, 173 patients (mean age $77 \pm 5$, range $70-94$; males $52 \%$ ) from a consecutive cohort of 236 (78\% inclusion rate), were randomized to MP $(n=86)$ or UC $(n=87)$ (Figure 1). Mean left ventricular ejection fraction (LVEF) was $33.4 \pm 10,7$ and mean NYHA class was $2.7 \pm 0.6$ (60,7\% NYHA 3-4). Previous MI was present in 92
$(53,2 \%)$ patients. All patients were treated with optimized evidence-based therapy (Betablockers 53,8\%, ACE-inhibitors or Angiotensin Receptor Blockers 91,9\%, Spironolactone 35.1\%).

Clinical correlates of Cognitive impairment. Cognitive impairment was present in 72 patients (41.6\%; $42,5 \%$ of usual care patients, $40.7 \%$ of MP patients $\mathrm{p}=\mathrm{ns}$ ). Table 1 shows the baseline characteristics of patients according to cognitive function. At multivariate logistic regression analysis the variables independently associated to CI were: age older than 80 years, education level $<5$ years, anemia and severe renal dysfunction. (Table 2). No association was found with blood pressure, LVEF or history of atrial fibrillation, hypertension and diabetes.

Outcomes. At two-year follow-up there were 59 deaths, 96 deaths and/or HF admissions, 113 allcause admissions and $77 \mathrm{HF}$ admissions. As a whole, MP intervention resulted in a significant reduction in deaths and/or HF admissions, and HF admissions [16].

When the entire cohort was analysed, cognitive impairment was independently associated to higher incidence of events (Table 3, undjusted chi-square test $\mathrm{p}$ values). At multivariate analysis, the risk of all-cause death increased more than 2-fold (HR $2,077[95 \%$ CI 1,097-3,931]) in patients with cognitive impairment; other independent predictors of mortality were: age, left ventricular ejection fraction $<20 \%$, hypotension, anemia, disability, and absence of Beta-blocker therapy (Table 4). Cognitive impairment was also independently associated to more frequent HF admissions (2,133[1,346-3,381]), more frequent death and/or HF admissions $(1,784[1,132-2,811])$ and all-cause admissions $(1,473[1,008-2,153]$ (Table 4).

Differences in treatment group (MP vs usual care) effects were then stratified and evaluated according to cognitive groups in a multivariate Cox model. Cognitive impairment was equally associated to all-cause death $(3,603$ [1,553-8,358], death/HF admissions $(2,029[1,200-3,432])$ and $\mathrm{HF}$ admissions $(2,474[1,406-4,353])$ but not to all-cause admissions. The assignment of patients with cognitive impairment to MP was associated to a significant reduction in HF admissions vs usual care $(0,503[0,253-0,999]$ (all interaction tests $\mathrm{p}=\mathrm{ns}$ ). Figure 2 shows the eventfree survival curves for the two treatment groups stratified according to cognitive status and compared by means of Log-rank Mantel-Cox tests. In both treatment groups, those with cognitive impairment appeared to have a worse prognosis, the risk of death and death/HF admission in MP patients being similar to those in usual care. By contrast, for HF admissions, a significant effect of both cognitive function and group assignment was observed, suggesting that the intervention is of benefit also in cognitively impaired patients. The risk of being admitted for $\mathrm{HF}$ was similar in patients with cognitive impairment enrolled in the MP and in cognitively intact patients in usual care. For all-cause admissions only a significant effect of group assignment was observed, with cognitively intact patients assigned to MP reporting a significant improvement (Figure 2). 
Tabella 1. - Clinical features according to presence or absence of cognitive impairment

\begin{tabular}{|c|c|c|c|}
\hline Variable & $\begin{array}{c}\text { Patients with } \\
M M S E \leq 24 \\
(n 72)\end{array}$ & $\begin{array}{c}\text { Patients with } \\
\text { MMSE }>24 \\
(\text { n 101 })\end{array}$ & $p$ \\
\hline Age (mean, years) & $79,4 \pm 5,7$ & $75,3 \pm 3,9$ & $<0,0001$ \\
\hline Older than 80 years & $28(38,9)$ & $14(13,9)$ & $<0,0001$ \\
\hline Men $(\%)$ & $27(37,5)$ & $63(62,4)$ & 0,001 \\
\hline Body mass Index $\left(\mathrm{Kg} / \mathrm{m}^{2}\right)$ & $23,5 \pm 4,3$ & $25,1 \pm 3,7$ & 0,016 \\
\hline Heart rate $(\mathrm{b} / \mathrm{min})$ & $75 \pm 12$ & $71 \pm 14$ & Ns \\
\hline Systolic blood pressure (mmHg) & $132 \pm 19$ & $131 \pm 21$ & ns \\
\hline NYHA III-IV class & $48(66,7)$ & $57(56,4)$ & $\mathrm{ns}$ \\
\hline Single marital status & $34(48,6)$ & $32(32,0)$ & 0,029 \\
\hline Living alone & $12(16,7)$ & $26(25,7)$ & Ns \\
\hline Low income level * & $16(22,5)$ & $7(7,1)$ & 0,004 \\
\hline Education level $<5$ yrs & $61(84,7)$ & $38(37,6)$ & $<0,0001$ \\
\hline $\begin{array}{l}\text { Heart Failure etiology } \\
\text { Ischemic } \\
\text { Hypertensive } \\
\text { Dilated } \\
\text { Valvular } \\
\text { Other/multiple }\end{array}$ & $\begin{array}{c}40(55,6) \\
5(6,9) \\
12(16,7) \\
11(15,3) \\
4(5,6)\end{array}$ & $\begin{array}{c}53(52,5) \\
17(16,8) \\
12(11,9) \\
14(13,9) \\
5(5)\end{array}$ & Ns \\
\hline $\mathrm{CKD} \geq \mathrm{IV}$ Stage $* *$ & $33(45,8)$ & $14(13,9)$ & $<0,0001$ \\
\hline Anemia \# & $50(69,4)$ & $48(47,5)$ & 0,004 \\
\hline Serum sodium & $138 \pm 4$ & $138+4$ & Ns \\
\hline Previous Myocardial infarction & $35(48,6)$ & $57(56,4)$ & Ns \\
\hline Permanent Atrial Fibrillation & $22(30,6)$ & $22(21,8)$ & Ns \\
\hline History of hypertension & $46(64)$ & $66(65)$ & ns \\
\hline History of diabetes mellitus & $26(36)$ & $29(29)$ & Ns \\
\hline History of COPD & $26(36,1)$ & $37(36,6)$ & Ns \\
\hline Charlson comorbidity index & $2,5 \pm 1,8$ & $1,9 \pm 1,3$ & $<0,01$ \\
\hline Left ventricular ejection fraction $(\%)$ & $33,7 \pm 11,7$ & $33,1 \pm 9,9$ & ns \\
\hline Beta-blockers & $32(44,4)$ & $61(60,4)$ & 0,038 \\
\hline ACE-inhibitors & $59(81,9)$ & $81(80,2)$ & Ns \\
\hline Angiotensin Receptor Blockers & $6(8,3)$ & $13(12,9)$ & Ns \\
\hline Spironolactone & $28(38,9)$ & $29(28,7)$ & Ns \\
\hline MMSE mean score & $21 \pm 3,6$ & $28 \pm 1,6$ & $<0,0001$ \\
\hline$\geq 1$ BADL Disability $\S$ & $32(44,4)$ & $13(28,9)$ & $<0,0001$ \\
\hline$\geq 2$ IADL Disability $\S$ & $53(73,6)$ & $29(28,7)$ & $<0,0001$ \\
\hline GDS 15 score $<6$ & $56(77,8)$ & $52(51,5)$ & $<0,0001$ \\
\hline Total direct costs of care & $4233,04 \pm 3397,00$ & $4744,92 \pm 3380,00$ & Ns \\
\hline
\end{tabular}

CKD: chronic kidney disease; BADL: Basic activities of daily living; IADL: Instrumental activities if daily living; MMSE: Mini mental state examination; GDS 15: 15-item Geriatric Depression Scale; * defined form the National Statistic Institute as an income $<600.00 €$ and $<900.00 €$ for one person and for a two-person family, respectively; $* *$ defined as an eGFR (glomerular filtration rate) $<30 \mathrm{ml} / \mathrm{min} / \mathrm{m} 2$; \# defined as Hemoglobin $<12 \mathrm{~g} / \mathrm{dl}$; § defined as impairment in at least one BADL or two IADL 
Table 2. Clinical variables significantly associated to cognitive impairment (Multivariate logistic regression analysis)

\begin{tabular}{lcccc}
\hline Variable & OR & \multicolumn{2}{c}{$\mathbf{9 5 , 0 \%}$ C.I. } & $P$ \\
\hline Age $>80$ years & 2,746 & 1,084 & 6,954 & 0,033 \\
\hline Education $<5$ yrs & 9,741 & 4,071 & 23,306 & $<0,0001$ \\
\hline Anemia & 2,380 & 1,113 & 5,091 & 0,025 \\
\hline CKD $\geq$ IV Stage & 4,641 & 1,841 & 11,700 & $<0,001$ \\
\hline
\end{tabular}

Table 3. Univariate outcome analysis at 2 year follow-up according to group assignment and cognitive status

\begin{tabular}{|c|c|c|c|c|c|c|c|c|c|}
\hline \multirow[t]{2}{*}{ Variable } & \multicolumn{3}{|c|}{$\begin{array}{l}\text { All Patients } \\
(\mathbf{n}=173)\end{array}$} & \multicolumn{3}{|c|}{$\underset{(n=86)}{M P}$} & \multicolumn{3}{|c|}{$\underset{(n=87)}{\mathbf{U C}}$} \\
\hline & $\begin{array}{c}M M S E \leq 24 \\
(n 72)\end{array}$ & $\begin{array}{c}M M S E>24 \\
(\text { (n 101) }\end{array}$ & $\mathbf{P}$ & $\begin{array}{l}M M S E \leq 24 \\
(n 35(40,7)\end{array}$ & $\begin{array}{l}M M S E>24 \\
\text { (n 51) }(59,3)\end{array}$ & $\mathbf{P}$ & $\begin{array}{c}M M S E \leq 24 \\
\quad(\text { (n 37) }\end{array}$ & $\begin{array}{c}M M S E>24 \\
(\text { (n 50) }\end{array}$ & $\mathbf{P}$ \\
\hline Deaths & $40(55,6)$ & $19(18,8)$ & $<0.001$ & $19(54,3)$ & $8(15,7)$ & $<0.0001$ & $21(56,8)$ & $11(22)$ & 0.001 \\
\hline $\begin{array}{l}\text { Death and or HF } \\
\text { admissions }\end{array}$ & $51(70,8)$ & $45(44,6)$ & $<0.001$ & $23(65,7)$ & $17(33,3)$ & 0.003 & $28(56)$ & $28(75,7)$ & 0,058 \\
\hline HF Admissions & $42(58,3)$ & $35(34,7)$ & 0.002 & $15(42,9)$ & $13(25,5)$ & ns & $27(73)$ & $22(44)$ & 0.007 \\
\hline $\begin{array}{l}\text { All cause } \\
\text { admissions }\end{array}$ & $51(70,8)$ & $62(61,4)$ & ns & $22(62,9)$ & $26(51)$ & ns & $29(78,4)$ & $36(72)$ & ns \\
\hline
\end{tabular}

Table 4. Multivariate predictors of outcome at 2-year follow-up (entire cohort) (Cox model)

\begin{tabular}{lllccc}
\hline Outcome & Variable & HR & \multicolumn{2}{c}{$\mathbf{9 5 \%}$ C.I. } & $\boldsymbol{p}$ \\
\hline All cause deaths & Age older $>80$ & 2,189 & 1,247 & 3,843 & 0,006 \\
& FEs 20\% & 2,532 & 1,210 & 4,195 & 0,004 \\
& SBP $<100$ mmHg & 4,722 & 1,930 & 11,551 & $<0,001$ \\
& Anemia & 3,494 & 1,800 & 6,780 & $<0,0001$ \\
& Beta-blockers & 0,459 & 0,258 & 0,817 & 0,008 \\
& BADL & 0,760 & 0,622 & 0,929 & 0,007 \\
& Cognitive impairment & 2,077 & 1,097 & 3,931 & 0,025 \\
\hline All cause deaths and/or & NYHA III-IV class & 3,745 & 2,177 & 6,444 & $<0,0001$ \\
heart failure admissions & SBP<100 mmHg & 2,294 & 1,156 & 4,553 & 0,018 \\
& Anemia & 1,814 & 1,156 & 2,870 & 0,011 \\
& Beta-blockers & 0,477 & 0,310 & 0,734 & $<0,001$ \\
& MP (vs UC) & 0,562 & 0,362 & 0,873 & $<0,01$ \\
& Cognitive impairment & 1,784 & 1,132 & 2,811 & 0,013 \\
\hline Heart failure admissions & NYHA III-IV class & 4,826 & 2,507 & 9,292 & $<0,0001$ \\
& Beta-blockers & 0,473 & 0,289 & 0,772 & 0,003 \\
& MP (vs UC) & 0,338 & 0,205 & 0,558 & $<0,0001$ \\
& Cognitive impairment & 2,133 & 1,346 & 3,381 & $<0,001$ \\
& Charlson index & 1,187 & 1,029 & 1,369 & 0,019 \\
\hline All-cause admissions & NYHA III-IV class & 2,171 & 1,372 & 3,436 & $<0,001$ \\
& Beta-blockers & 0,459 & 0,306 & 0,690 & $<0,0001$ \\
& MP(vs UC) & 0,456 & 0,305 & 0,682 & $<0,0001$ \\
& Cognitive impairment & 1,473 & 1,008 & 2,153 & 0,045 \\
& Charlson index & 1,159 & 1,021 & 1,315 & 0,023 \\
\hline
\end{tabular}

Estimation of direct costs. The mean two-year costs of care were similar in patients with and without cognitive impairment $(4233,04 \pm 3397,00$ vs 4744, 92 $\pm 3380,00 ; \mathrm{p}=\mathrm{ns}$ ) (Table 1). The total cost in the MP group was $€ 4538,97 \pm 3629,64$ in patients with cognitive impairment and $€ 3740,72+3213,92$ in patients without $(\mathrm{p}=\mathrm{ns})$. The total cost of care in usual care group was $€ 4939,78 \pm 3163,69$ in patients with cognitive impairment and $4836,21 \pm 3342,73$ in patients without $(\mathrm{p}=\mathrm{ns})$. 


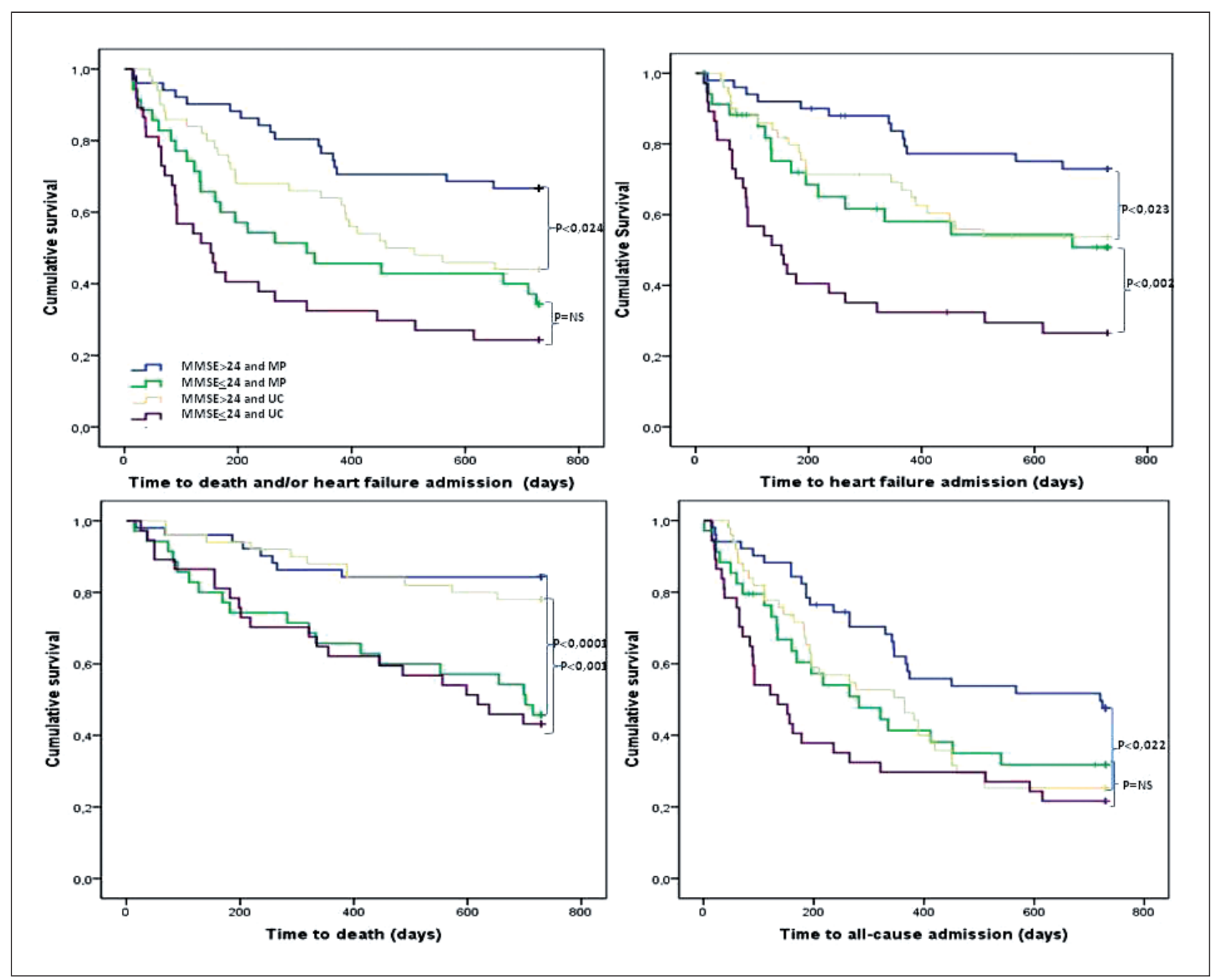

Figure 2. Kaplan-Meyer event-free survival curves stratified for the two treatment groups and cognitive status. For death and/or HF admission and heart failure admissions, a significant effect of both cognitive function and group assignment was observed (Upper panels). By contrast, risk of death in MP patients was similar to those in usual care (Left lower panel). For all-cause admissions only a significant effect of group assignment was observed, with cognitively intact patients assigned to MP reporting a significant improvement(Right lower panel).

\section{Discussion}

Available evidence [6-8] suggests that HF MPs are a consistent way to provide appropriate, more comprehensive and cost-effective care. In older HF patients many re-admissions can be often attributed to co-morbidities, behavioural and social factors rather than to deteriorating cardiac function or an incident cardiac event. Older patients are also less likely to receive recommended therapies, in part because clinical trials of HF therapy have ignored outcomes of importance to this population, including comorbidities and impaired cognitive function [2, 4, 5]. Cognitive impairment represents a risk factor for low adherence to treatments, disability, hospitalization and death. Nevertheless, it has been rarely assessed in previous studies on HF [11-14], and it is still not well established if reported models of intervention are effective in these patients [6-8, 23].

The results of this perspective study confirm that cognitive impairment is a prevalent condition associated to a worse prognosis, and suggest that a hospital-based, integrated MP may improve outcomes in these patients through reduction in HF hospital admission.
Cognitive impairment is significantly and independently associated to a higher risk of adverse outcomes also in patients in optimised therapy followed by specialists, suggesting that attention for this noncardiovascular comorbidity seems to be essential for optimizing treatment and improve prognosis. It is of note that cognitive impairment, as well as other variables such disability or frailty are seldom included in statistical models and patient predictors of readmission for HF [24].

Previous published trials on MPs rarely examined the relative effectiveness of the intervention among cognitively impaired patients. In HF patients, cognitive impairment contributes with social and behavioral problems in making difficult to engage in self-care, in decreasing compliance to careplan and prescribed therapy in a safe and effective manner and, consequently, increasing hospital admissions [14]. Moser et al. pointed out the role of cognition in their conceptual framework of factors affecting decision making and subsequent self-care in patients with HF [25]. Cognitive impairment may contribute to failed self-care in two ways: through deficits in memory and attention that may impair treatment adherence, because of forgetfulness and 
poor learning ability, and through problems in executive functions, information processing and decision making in complex situations, such as early recognition and interpretation of symptoms and seeking care [26].

McLennan et al, reported that a nurse-lead home-based multidisciplinary program may not improve the prognosis of patients with mild cognitive impairment. We observed similar findings with regards to all-cause mortality, but not HF readmissions or the combined end-point. However the model of intervention and the follow-up duration were significantly different than that used in the present study [15]. Our findings clearly demonstrate that enrolment in a MP is associated with a clinically important and sustained decline in $\mathrm{HF}$ hospital admissions over a 2-year intervention period in comparison to usual care, also in patients with mild-to-moderate cognitive impairment. The risk of being admitted for HF was similar in patients with CI enrolled in the MP and in cognitively intact patients in usual care (Figure 2). Moreover, economic analysis suggests that MP does not increase direct costs of care also in patients with mild-to-moderate CI.

A possible reason for the favourable effect of a MP on HF admissions is that cognitively impaired patients may need intensive education program and follow-up through office or home visits or telephone contacts to prevent adherence issues, a major cause of re-hospitalization. Written and given verbally care instructions and medication or dietary changes are necessary because of patient difficulties with information requiring attention, learning, and memory functions. Our MP provided clinical monitoring and a structured and continuing education to the patient and their caregiver according to guidelines [17, 27].

Thus, a rapid assessment of cognitive function, particularly in patients of advanced age, with poor social status, anemia or renal dysfunction, may lead to a more intensive follow-up and tailored education in order to reduce the risk of non adherence, reduced self-care and re-hospitalisation.

Limitations. The strengths of the present study are the accuracy of clinical and instrumental evaluation, the clinical and hemodynamical stability at the time of assessment, the optimisation of evidence-based treatments and the completeness and duration of follow-up. However, it deserves several limitations.

First, this study excluded those patients with severe cognitive impairment in which disability, lowcompliance and incidence of events in the final stages of the syndrome are extremely high, more likely to follow-up with intensive, home-based programs [6-8, 22, 23]. Thus, it is likely that not all community-dwelling older patients will benefit from such a model of intervention.

Second, cognitive function, disability and quality of life are complex and time consuming to measure. In our study cognitive evaluation was performed using a limited number of tests instead of an extensive and more accurate but also time-consuming battery. Moreover, the MMSE has poor sensitivity and specificity versus memory dysfunction, and in the detection of very mild forms of cognitive impairment $[13,28]$. Thus, a screening instrument for easy use in clinical practice more sensitive than MMSE needs to be identified. However, the tests included in the present study were selected from those available on the basis of recognised reliability [11] as well as usability in a realworld ambulatory setting, where time and personnel resources are of importance. In this view, this study suggest that also a short minimal data set is useful and applicable for screening purposes in a HF clinic.

In conclusion, in spite of optimized therapy and specialist care, $\mathrm{CI}$ is very common and associated to worse prognosis in $\mathrm{HF}$ and hospital-based, integrated MP appears to be equally effective in mildto-moderately cognitively impaired patients through reduction in HF hospital admission.

These results - if confirmed - may have clear implications in clinical practice and can be a useful contribution for an optimal allocation of healthcare resources [27]. A multidisciplinary approach is necessary to deal with the complexity of this clinical syndrome and aassessment of cognitive function, even by the simple screening tests used in this study, should be part of the baseline evaluation $[29,30]$.

Contributors: GiP, DDS, ADL, LT and GC were involved in planning the study and wrote the study protocol and the manuscript. GiP and DDS prepared summaries of all hospital admissions for the end point committee (GM, LT, GC). GiP and DDS supervised the activity of the enrolling HF clinics and provided medical support. MDT, ST and LM collected and assembled the clinical data. GB and GiP carried out the statistical analysis. All investigators were involved in reviewing the manuscript.

Supported in part by the nonprofit organization ADRIANO-Italian Association for Research on Cardiac Disease in Older Patients (Program ADDisease management program 001-2004).

\section{Riassunto}

Background: Il deterioramento cognitivo complica frequentemente lo Scompenso Cardiaco (SC) ed è associato con un aumento di mortalità e morbilità. Studi precedenti riportano che programmi multidisciplinari domiciliari possono non migliorare la prognosi di questo gruppo di pazienti ad alto-rischio. Nel seguente studio abbiamo analizzato l'efficacia di un programma integrato multidisciplinare (MP) basato sull' ospedale in pazienti con DC.

Metodi: Abbiamo arruolato pazienti in condizioni cliniche stabili non istituzionalizzati di età $>70$

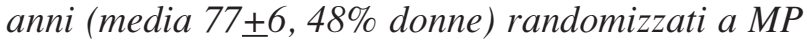
$(n=86)$ o cure convenzionali $(U C)(n=87)$. Lo stato cognitivo è stato valutato mediante Mini Mental State Examination (MMSE).

Risultati: Deficit cognitivo $(M M S E \leq 24)$ era presente nel $41.6 \%$ (42,5\% UC vs $40.7 \% \bar{M} P$ p=ns) e si associava indipendentemente a: età, scolarità 
$<5$ anni, anemia e disfunzione renale severa. A 2 anni di follow-up, 59 pazienti sono morti (31.4\%) senza differenze significative tra $i$ gruppi. All' analisi multivariata, nell' intera coorte, il deficit cognitivo era associato a morte (HR 2,077[95\% CI 1,097$3,931])$, ricoveri per $S C(2,133[1,346-3,381])$, mortelricoveri per SC $(1,784[1,132-2,811])$ e ricoveri per tutte le cause $(1,473[1,008-2,153]$. Stratificando per gruppi di intervento, il deficit cognitivo era ancora associato alla mortalità totale $(3,603$ [1,5538,358], morte/ricoveri per SC (2,029[1,200-3,432]), ricoveri per SC $(2,474[1,406-4,353])$. I pazienti con deficit cognitivo assegnati a MP riportavano una riduzione significativa di ricoveri per $S C$ $(0,503[0,253-0,999]$ (test di interazione $p=n s)$.

Conclusioni: Il deficit cognitivo è molto comune e associato a prognosi peggiore nello SC e un programma multidisciplinare sembra migliorare la prognosi in questi pazienti attraverso una riduzione dei ricoveri per $S C$.

\section{LIST OF ABBREVIATIONS}

CI: cognitive impairment

$\mathrm{HF}$ : heart failure

MP: multidisciplinary program

UC: usual care

MMSE: Mini Mental State Examination

HR: hazard ratio

NYHA: New York Heart Association

BADL: basic activities of daily living

IADL: instrumental activities of daily living

GDS: Geriatric Depression Scale

NHS: National Health System

SD: standard deviation

CI: confidence interval

LVEF: left ventricular ejection fraction

MI: myocardial infarction

eGFR: glomerular filtration rate

Acknowledgments: We thank Attilia Caroselli, RN, Lino Fabrizi, RN, Luca Guerrieri, RN, Marina Rotoloni, RN, Francesca Stefanelli, RN, and Rita Venusti, $\mathrm{RN}$, for their nursing assistance in the education and evaluation of patients.

We also thank the Physicians and Nurses working in the Cardiology units and local General Practitioners for participation in the study protocol and for allowing us to follow-up their patients.

\section{References}

1. Senni M, Tribouilloy CM, Rodeheffer RJ et al. Congestive heart failure in the community: trends in incidence and survival in a 10-year period. Arch Intern Med 1999; 159: 29-34

2. Pulignano G, Del Sindaco D, Tavazzi L et al. Clinical features and outcomes of elderly outpatients with HF followed up in hospital cardiology units: data from a large nationwide cardiology database (Italian Network on Congestive Heart Failure [IN-CHF] Registry). Am Heart $J$ 2002; 143: 45-55.

3. Havranek EP, Masoudi FA, Westfall KA, Wolfe P, Ordin DL, Krumholz HM. Spectrum of heart failure in older patients: results from the National Heart Failure project. Am Heart J 2002; 143: 412-7.

4. Pulignano G, Del Sindaco D, Di Lenarda A, Sinagra G. The evolving care of the elderly with heart failure: from the 'high-tech' to the 'high-touch' approach. $J$ Cardiovasc Med (Hagerstown) 2006; 7: 841-846.

5. Rich MW. Heart failure in the oldest patients: the impact of comobid conditions. AJGC 2005; 14: 134-41.

6. Phillips CO, Wright SM, Kern DE, Singa RM, Shepperd $\mathrm{S}$, Rubin HR. Comprehensive discharge planning with postdischarge support for older patients with congestive heart failure. A meta-analysis. JAMA 2004; 291: 1358-67.

7. Gonseth J, Guallar-Castillon P, Banegas JR, RodriguezArtalejo F. The effectiveness of disease management programmes in reducing hospital re-admission in older patients with heart failure: a systematic review and metaanalysis of published reports. Eur Heart $J$ 2004; 25 : $1570-95$.

8. Roccaforte R, Demers C, Baldassarre F, Teo KK, Yusuf S. Effectiveness of comprehensive disease management programmes in improving clinical outcomes in heart failure patients. A meta-analysis. Eur J Heart Fail 2005; 7 : 1133-44.

9. Riegel B, Carlson B, Glaser D, Hoagland P. Which patients with heart failure respond best to multidisciplinary disease management? J Card Fail 2000; 6: 290-9.

10. Clark A, Thompson DR. What heart failure programme works best? Wrong question, wrong assumptions. Eur J Heart Fail 2010; 12, 1271-1273.

11. Vogels RLC, Oosterman JM, van Harten B et al. Profile of Cognitive Impairment in Chronic Heart Failure. Journal of the American Geriatrics Society 2007; 55(11): 1764-1770.

12. Zuccala G, Pedone C, Cesari M et al. The effects of cognitive impairment on mortality among hospitalized patients with heart failure. Am J Med 2003 Aug 1; 115(2): 97-103.

13. Antonelli-Incalzi R, Trojano L, Acanfora D et al. CHF Italian StudyInvestigators. Verbal memory impairment in congestive heart failure. Journal of Clinical and Experimental neuropsychology 2003; 25: 14-23.

14. Dickson VA, Tkas N, Riegel B. Cognitive influences on self-care decision making in persons with heart failure. Am Heart J 2008; 154: 424-431.

15. McLennan SN, Pearson SA, Cameron J, Stewart S. Prognostic importance of cognitive impairment in chronic heart failure patients: Does specialist management make a difference? Eur J Heart Fail 2006; 8: 494-501

16. Del Sindaco D, Pulignano G, Minardi G, et al. Two-year outcome of a prospective, controlled study of a disease management programme for elderly patients with heart failure. J Cardiovasc Med 2007; 8: 324-9.

17. Task Force for the Diagnosis and Treatment of Chronic Heart Failure of the European Society of Cardiology. Guidelines for the diagnosis and treatment of chronic heart failure. Eur Heart $J$ 2001; 22: 1527-1560.

18. Katz S, Ford AB, Moskowitz RW, Jackson BA, Jaffe MW. Studies of illness in the aged: the index of ADL: a standardized measure of biological and psychosocial function. $J$ Am Med Assoc 1963; 185: 914-919.

19. Lawton MP, Brody EM, Assessment of older people: self-maintaining and instrumental activities of daily living. Gerontologist 1969; 9: 179-186.

20. Folstein MF, Folstein SE, McHugh PR. 'Mini-Mental State': a practical method for grading the cognitive state of patients for the clinician. J Psychiatr Res 1975; 12: 189-198.

21. Sheikh JI, Yesavage JA. Geriatric Depression Scale (GDS): recent evidence and development of a shorter version.In: Brink TL. editor. Clinical gerontology: a guide to assessment and intervention. New York: Haworth Press; 1986. pp. 165-173.

22. Cockcroft DW, Gault MH. Prediction of creatinine clearance from serum creatinine. Nephron 1976; 16: 31-41.

23. Yu DS, Thompson DR, Lee DT. Disease management programmes for older people with heart failure: crucial characteristics which improve post-discharge outcomes. Eur Heart J 2006; 27: 596-612. 
24. Ross JS, Mulvey GK, Stauffer B et al. Statistical Models and Patient Predictors of Readmission for Heart Failure. A Systematic Review. Arch Intern Med 2008; 168(13): 1371-1386.

25. Moser DK, Watkins JF. Conceptualizing self-care in heart failure: a life course model of patient characteristics. J Cardiovasc Nurs 2008; 23: 205-218.

26. Taylor J, Scott DJ. Chronic heart failure and cognitive impairment: co-existence of conditions or true association? Eur J Heart Fail 2002; 4: 7_936.

27. Hauptman PJ, Rich MW, Heidenreich PA, et al. The Heart Failure Clinic: A Consensus Statement of the Heart
Failure Society of America. J Cardiac Fail 2008; 14: $801 \mathrm{e} 815$

28. Tombaugh TN, McIntyre NJ. The mini-mental state examination: a comprehensive review. Am Geriatr Soc 1992; 40: 922-35.

29. Cohen MB, Mather PJ. A review of the association between congestive heart failure and cognitive impairment. Am J Geriatr Cardiol 2007 May-Jun; 16(3): 171-4

30. Heckman GA, Patterson CJ, Demers C, St Onge J, Turpie ID, McKelvie RS. Heart failure and cognitive impairment: challenges and opportunities. Clin Interv Aging 2007; 2(2): 209-18. 